\title{
Contact Lens-associated Keratitis by Elizabethkingia meningoseptica
}

${ }^{1}$ Manisha Kharbanda, ${ }^{2}$ Parag Bagad, ${ }^{3}$ Sunanda Dey

\begin{abstract}
Elizabethkingia meningoseptica is a Gram-negative bacillus. It is known for causing neonatal meningitis and pneumonia, sepsis in case of immunocompromised patients. It can be found in hospital and natural environments. It may exist in freshwater, salt water, and soil. It was previously known as Flavobacterium and Chryseobacterium meningosepticum.

Contact lens-induced keratitis with E. meningoseptica which we are reporting is the second case report till date. It has striking resemblance with the case that was published earlier, with minimal difference in presentation, but significant difference in the antimicrobial sensitivity. Most importantly, we also found this infection in healthy individual with no underlying disease.

Thus, we strongly support the case report that was published earlier, and though rare, this microbe should be considered as potential pathogen for contact lens-related keratitis, and increasing resistance to the antimicrobial agents should also be kept in mind.
\end{abstract}

Keywords: Contact lens, Elizabethkingia meningoseptica, Keratitis.

How to cite this article: Kharbanda M, Bagad P, Dey S. Contact Lens-associated Keratitis by Elizabethkingia meningoseptica. Int J Recent Surg Med Sci 2016;2(2):102-105.

Source of support: Nil

Conflict of interest: None

\section{INTRODUCTION}

Contact lens-related microbial keratitis is rising and is likely to cause severe, vision-threatening infections. ${ }^{1}$ Bacterial keratitis is a vision-threatening contact lens complication. Corneal epithelial damage owing to hypoxia and minor trauma is equally important as bacterial adherence to the contact lens surface. Wearing soft contact lenses poses more risk than rigid gas-permeable and other types. ${ }^{2,3}$ Wearing and sleeping with contact lens

\footnotetext{
${ }^{1}$ Consultant, ${ }^{2}$ Final Year DNB, ${ }^{3}$ Microbiologist

1,2Department of Ophthalmology, Rotary Narayana Nethralaya Kolkata, West Bengal, India

${ }^{3}$ Department of Microbiology, SRL Diagnostic Centre, Kolkata West Bengal, India

Corresponding Author: Manisha Kharbanda, Consultant Department of Ophthalmology, Rotary Narayana Nethralaya Kolkata, West Bengal, India, Phone: +918100981411, e-mail: dr.paragbagad@yahoo.in
}

are the major risk factors. Infection occurs due to poor lens hygiene; ${ }^{15}$ however, it may occur despite meticulous lens care and with daily disposable lenses. ${ }^{2}$

A contaminated contact lens case may act as a reservoir for microorganisms that may compromise contact lens wear and lead to sight-threatening events. ${ }^{4}$

Elizabethkingia meningoseptica (earlier known as Flavobacterium meningosepticum and then Chryseobacterium meningosepticum) is a nosocomial, nonfermenting Gramnegative bacillus with intrinsic resistance to many antibiotics, which are usually administered in intensive care settings. ${ }^{5}$

In addition, it carries many antimicrobial resistance genes, as well as has potential to form biofilms and survive in a moist environment or in water sources including tap water, making it an important organism in many intensive care environments. ${ }^{6,7}$

Elizabethkingia meningoseptica is a rare but serious cause of neonatal meningitis and sepsis, as well as an increasingly important pathogen in health care environments; its role as a cause of ocular pathology is not well known. ${ }^{5}$

However, single case of contact lens-related microbial keratitis with E. meningoseptica has been reported till date with many similarities with our case but significant difference in antimicrobial sensitivity testing. ${ }^{8}$

\section{CASE REPORT}

A 21-year-old female patient visited our hospital due to left ocular grittiness. She had been wearing soft contact lenses for 8 months, approximately 8 hours per day. She had no medical history of ocular injury, surgery, or treatment with either ophthalmic or systemic medications. On our initial examination, visual acuity was 6/6 in the right eye and 6/9 in the left eye. Slit-lamp examination revealed hyperemia of the left bulbar conjunctiva, two corneal epithelial defects sized $0.5 \times 0.5 \mathrm{~mm}$ along with intrastromal infiltration, which was of same size as that of epithelial defect. However, neovascularization was absent. Corneal sensitivity was within normal limit. The lens, vitreous body, and retina were all normal.

The patient was treated as follows: Unpreserved moxifloxacin eye drop $0.3 \%, 2 \%$ homatropine three times a day, and $1 \%$ carboxymethyl cellulose six times a day. 


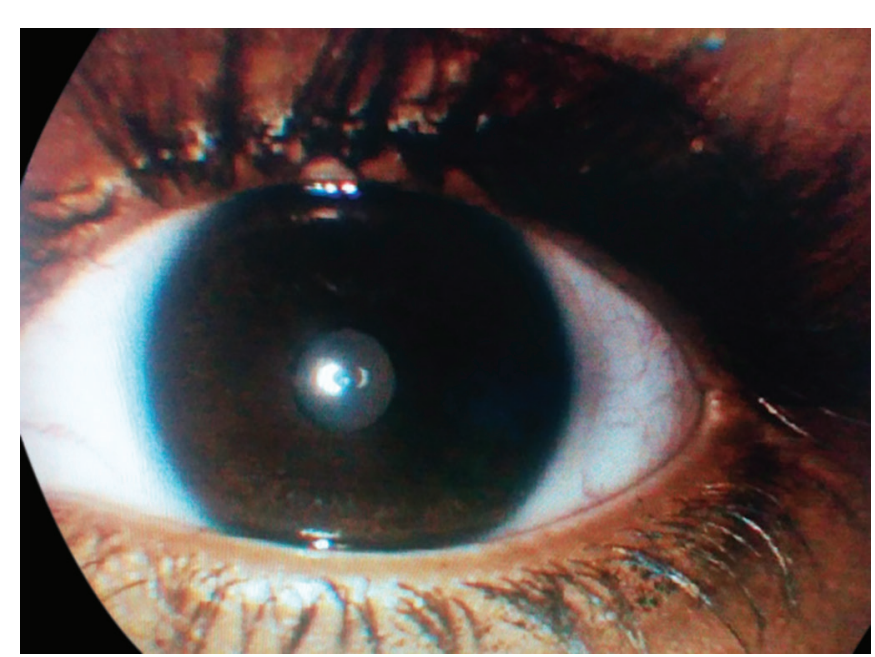

Fig. 1: Depiction of the remarkable improvement after 2 days of treatment

The patient was firstly examined at the peripheral center, so anterior segment photography and corneal scraping were not being performed.

Two days later, patient was called for the follow-up at our main tertiary care center. The contact lens and its case were then sent for bacteriological test. ${ }^{16}$ Patient was symptomatically better. The corneal epithelial defect was nearly healed, and the corneal intrastromal infiltration was also decreased significantly (Fig. 1).

After the initial bacterial culture, bacteria were inoculated and cultured in MacConkey agar at an intermediate level, in specimens (Fig. 2A). Gram staining revealed pink rods suggestive of Gram-negative bacilli (Fig. 2B). Elizabethkingia meningoseptica was identified by using the VITEK 2 compact instrument (Biomerieux). Elizabethkingia meningoseptica was sensitive to fluoroquinolones, trimethoprim/sulfamethoxazole, but resistant to gentamicin, piperacillin/tazobactam, and amikacin (Table 1).

The patient was thus continued on moxifloxacin $(0.3 \%)$ and $1 \%$ carboxymethyl cellulose four times a day.
However, 2\% homatropine was discontinued. Complete reepithelialization of the lesion was observed on the 7 th day after treatment. After 2 weeks, the patient was being treated only with $1 \%$ carboxymethyl cellulose four times a day. Her final corrected visual acuity in the right eye was $6 / 6$.

\section{DISCUSSION}

Elizabethkingia meningoseptica is found in hospital and natural environments, such as soil and seawater. In hospital settings, it is seen in water supplies, the saline solution, disinfectants, and medical devices. The bacterium is an aerobic, Gram-negative rod, and it may cause pneumonia and sepsis in immunologically compromised and postoperative adults. ${ }^{9}$

So far, only one case ${ }^{8}$ of soft contact lens-related keratitis with E. meningoseptica as a sole cause with striking resemblance with our case has been reported.

Similarities include the young age group, healthy individual, history of prolong contact lens wear, epithelial erosion, and intrastromal infiltration; however, in our case, neovascularization of cornea was not observed, which was present in the earlier case.

Elizabethkingia meningoseptica was sensitive to ciprofloxacin, trimethoprim/sulfamethoxazole, levofloxacin, gentamicin, piperacillin/tazobactam, and amikacin in the earlier case; however, in our case, E. meningoseptica was sensitive to fluoroquinolones and trimethoprim/ sulfamethoxazole, but resistant to gentamicin, piperacillin/tazobactam, and amikacin (Table 1).

Few cases of keratitis associated with E. meningoseptica have been described earlier.

In the first case, keratitis was caused by polymicrobial etiology, in which a 14-year-old cosmetic contact lens user presented with a paracentral corneal ulcer in her right eye. She had bought the contact lenses online. The cultures from corneal scrapings and contact lenses
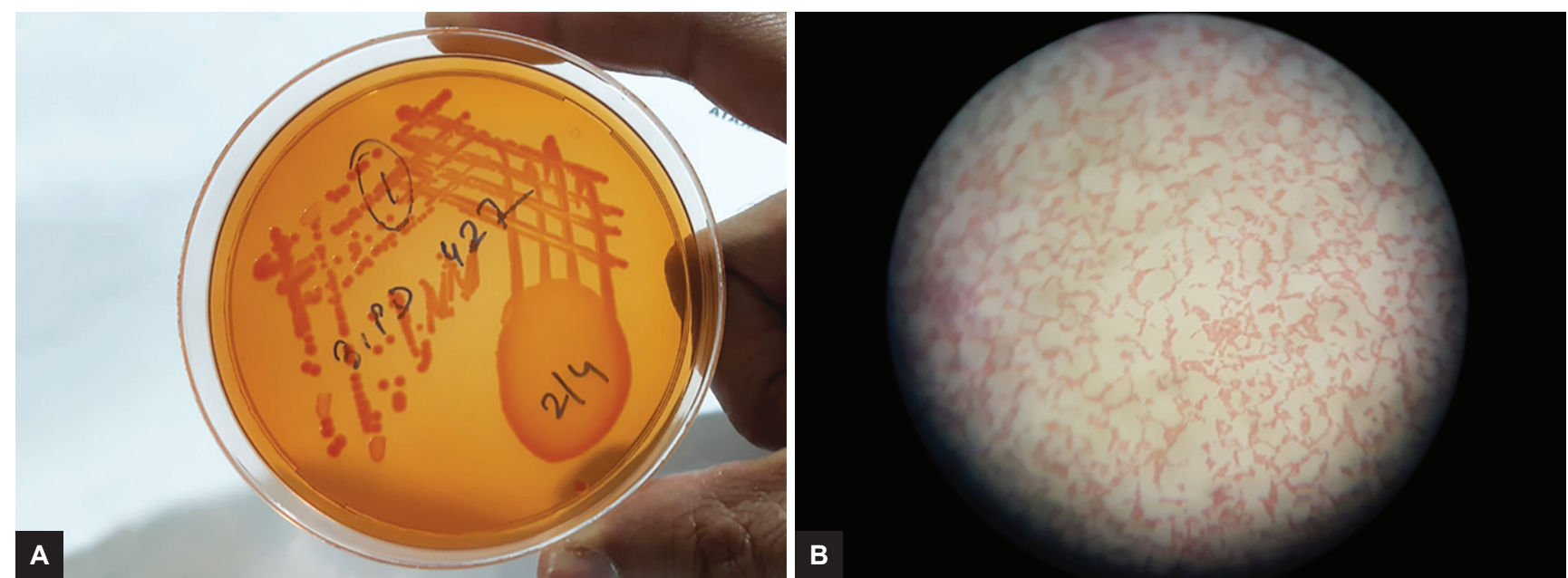

Figs 2A and B: (A) Multiple colonies on MacConkey's agar; and (B) Gram stain showing Gram-negative bacilli 
Table 1: Antibiotic sensitivity test

\begin{tabular}{|c|c|c|}
\hline First-line antibiotic & $\begin{array}{l}\text { Result MIC } \\
(\mu g / m L)\end{array}$ & Interpretation \\
\hline Ampicillin & $\geq 32$ & Resistant \\
\hline Gentamicin & $\geq 16$ & Resistant \\
\hline Nitrofurantoin & $\geq 512$ & Resistant \\
\hline Trimethoprim-sulfamethoxazole & 40 & Sensitive \\
\hline \multicolumn{3}{|l|}{ Second-line antibiotics } \\
\hline Amoxicillin clavulanate & $\geq 32$ & Resistant \\
\hline Piperacillin tazobactam & $\geq 128$ & Resistant \\
\hline Ciprofloxacin & 0.50 & Sensitive \\
\hline Cefuroxime & $\geq 64$ & Resistant \\
\hline Ceftriaxone & $\geq 64$ & Resistant \\
\hline Cefoperazone sulbactam & 32 & Intermediate \\
\hline Amikacin & $\geq 64$ & Resistant \\
\hline Imipenem & $\geq 16$ & Resistant \\
\hline Meropenem & $\geq 16$ & Resistant \\
\hline \multicolumn{3}{|l|}{ Supplemental antibiotics } \\
\hline Nalidixic acid & 8 & Sensitive \\
\hline Tigecycline & 4 & Intermediate \\
\hline
\end{tabular}

Specimen - Contact lens and contact lens case; aerobic culture: (method - colorimetric): Positive; organism: E. meningoseptica

showed heavy growth of $C$. meningosepticum and Delftia acidovorans. The treatment with topical ciprofloxacin and fortified gentamicin was effective, and the infection resolved with corneal scar after 5 weeks. ${ }^{9}$

The second case too had polymicrobial etiology, in which the patient presented with bilateral simultaneous infectious keratitis secondary to contact lens wear. In this case, a 21-year-old soft contact lens wearer visited the emergency department with a 3-day history of pain, redness, decreased vision, photophobia, and tearing in both eyes. On examination, a central corneal ulcer was identified with hypopyon in both eyes. The cultures from corneal scrapings of both eyes, the contact lenses, and the contact lens solution showed heavy growth of Pseudomonas aeruginosa, Alcaligenes species, and F. meningosepticum. ${ }^{10}$

The third case was posttraumatic keratitis caused by Elizabethkingia meningosepticum.

A 45-year-old woman presented with keratitis, which had developed following nonpenetrating eye trauma from a tree branch. Cultures from a corneal smear demonstrated heavy growth of E. meningosepticum. Patient responded well to combination of topical moxifloxacin and topical trimethoprim/polymyxin B. Corneal infection resolved within 2 months, but a central corneal scar and vascularization remained. ${ }^{11}$

In the fourth case, a 48-year-old man presented with a corneal ulcer in his right eye and was treated with ofloxacin and fortified bacitracin ophthalmic solution. Cultures grew $C$. meningosepticum and the infection resolved. Patient had no significant comorbidities. ${ }^{12}$

The fifth case had developed massive lipid keratopathy after E. meningosepticum keratitis.
A 52-year-old female presented with complaints of pain, redness, watering, and blurred vision in her left eye for 6 days. Patient already had undergone radiotherapy for mucoepidermoid carcinoma of the maxillary antrum, which was later complicated by radiotherapyassociated dry eye syndrome in her left eye. At the time of presentation, her best-corrected visual acuity was hand movements close to face in the left eye. Slit-lamp examination of the left eye showed a small infiltrate $1.4 \times$ $1.2 \mathrm{~mm}$ in size with an overlying epithelial defect. Elizabethkingia meningosepticum was isolated on broth. Good clinical response was observed with hourly $5 \%$ vancomycin eye drops.

At the end of 8 weeks, slit-lamp examination of the left eye revealed a corneal scar with mild lipid deposition at the site of previous corneal infiltrate, which increased tremendously over the next 4 weeks. ${ }^{13}$

In our case, we observed ocular infection with E. meningoseptica in an eye with no significant comorbidities. We suspected the contact lens-induced keratitis; thus, microbiological examinations were performed on the contact lenses and the contact lens cases. The growth of E. meningoseptica was confirmed; this made it possible to ascertain that the contact lenses were the direct cause of keratitis. To the best of our knowledge, E. meningoseptica has been isolated exclusively only once in patients with contact lens-related microbial keratitis, in the past.

Contact lens-induced corneal hypoxia raises the chance of corneal infection by jeopardizing corneal epithelial integrity, affecting wound healing, and increasing binding of bacteria to corneal epithelial cells. Similarly, the use of contaminated saline solution, inappropriate management of the contact lenses, or direct contact with contaminated water increase the risk of corneal infection. Thus, proper lens hygiene should be maintained to prevent corneal infection. Contact lens users need to wash their hands before handling their lenses, ${ }^{14}$ keep their lens cases clean at all times, replace their cases at regular intervals, and replace their soft contact lenses at least every 3 months.

Contact lens-induced keratitis with E. meningoseptica which we are reporting is the second case reported till date. It has got striking resemblance with the case that was published earlier, ${ }^{8}$ with minimal difference in presentation, but significant difference in antimicrobial sensitivity. Most importantly, we also found this infection in healthy individual with no underlying disease.

Thus, we strongly support the case report that was published earlier, and though rare, this microbe should be considered as potential pathogen for contact lens-related keratitis, and increasing resistance to the antimicrobial agents should also be kept in mind. 


\section{REFERENCES}

1. Cheung N, Nagra P, Hammersmith K. Emerging trends in contact lens-related infections. Curr Opin Ophthalmol 2016 Jul;27(4):327-332.

2. Bowling B. Cornea. In: Gabeedy, R.; Cook, L.; Leonard, J. Kanski's clinical ophthalmology: a systemic approach. 8th ed. Elsevier; 2016. p. 173.

3. Nagachandrika T, Kumar U, Dumpati S, Chary S, Mandathara PS, Rathi VM. Prevalence of contact lens related complications in a tertiary eye centre in India. Cont Lens Anterior Eye 2011 Dec;34(6):266-268.

4. Wu YT, Willcox M, Zhu H, Stapleton F. Contact lens hygiene compliance and lens case contamination: a review. Cont Lens Anterior Eye 2015 Oct;38(5):307-316.

5. Young SM, Lingam G, Tambyah PA. Elizabethkingia Meningoseptica endogenous endophthalmitis - a case report. Antimicrob Resist Infect Control 2014;3:35.

6. Liu LY, Seo J, McCanna DJ, Subbaraman LN, Jones LW. Assessment of biofilm formation of E. meningoseptica, D. acidovorans, and S. maltophilia in lens cases and their growth on recovery media. Cont Lens Anterior Eye 2016 Apr;39(2):117-123.

7. Willcox MD. Microbial adhesion to silicone hydrogel lenses: a review. Eye Contact Lens 2013 Jan;39(1):61-66.

8. Yang YS, Chun JW, Koh JW. Keratitis with Elizabethkingia meningoseptica occurring after contact lens wear: a case report. Korean J Ophthalmol 2013 Apr;27(2):133-136.
9. Ray M, Lim DK. A rare polymicrobial keratitis involving Chryseobacterium meningosepticum and Delftia acidovorans in a cosmetic contact lens wearer. Eye Contact Lens 2013 Mar;39(2):192-193.

10. Ali NA, Reddy SC. Bilateral simultaneous infectious keratitis secondary to contact lens wear: an unusual case report with rare organisms. Eye Contact Lens 2007 Nov;33(6 Pt 1): 338-340.

11. Erdem E, Abdurrahmanoglu S, Kibar F, Yagmur M, Koksal F, Ersoz R. Posttraumatic keratitis caused by Elizabethkingia meningosepticum. Eye Contact Lens 2013 Sep;39(5):361-363.

12. Bloom AH, Perry HD, Donnenfeld ED, Davis RG. Chryseobacterium meningosepticum keratitis. Am J Ophthalmol 2003 Aug;136(2):356-357.

13. Li EY, Jhanji V. Massive lipid keratopathy after Elizabethkingia meningosepticum keratitis. Cont Lens Anterior Eye 2014 Feb;37(1):55-56.

14. McMonnies CW. Hand hygiene prior to contact lens handling is problematical. Cont Lens Anterior Eye 2012 Apr;35(2): 65-70.

15. Thakur DV, Gaikwad UN. Microbial contamination of soft contact lenses and accessories in asymptomatic contact lens users. Indian J Med Res 2014 Aug;140(2):307-309.

16. Das S, Sheorey H, Taylor HR, Vajpayee RB. Association between cultures of contact lens and corneal scraping in contact lens related microbial keratitis. Arch Ophthalmol 2007 Sep;125(9):1182-1185. 